\title{
RACIONALIDAD Y ACCIÓN NO REFLEXIVA. EL DEBATE DREYFUS-MCDOWELL
}

\author{
JuAn Pablo Hernandez Betancur \\ Pontificia Universidad Javeriana \\ hernandez-juan@javeriana.edu.co
}

RESUMEN: El debate reciente iniciado por Hubert Dreyfus y John McDowell ha llamado la atención sobre la relación entre racionalidad y acción no reflexiva. En este artículo propongo una forma de especificar el desacuerdo entre quienes llamaré intelectualistas y antiintelectualistas. A la luz de esta propuesta arguyo que el principal argumento antiintelectualista sólo tiene éxito si se acepta al menos uno de tres presupuestos implícitos que están lejos de ser autoevidentes y cuya verdad es puesta en duda por fenómenos familiares. Termino con consideraciones que sugieren que el intelectualista ha quedado en una mejor posición argumentativa.

PALABRAS CLAVE: intelectualismo, razones para la acción, contenido no conceptual, fenomenología, desempeño absorto

SUMMARY: The recent debate initiated by Hubert Dreyfus and John McDowell has called attention on the relation between rationality and unreflective action. In this paper I propose a form of specifying the disagreement between intellectualists and anti-intellectualists, as I will call them. In light of this proposal I argue that the main anti-intelectualist argument can only succeed if at least one of three implicit presuppositions is granted. These presuppositions are far from being self-evident and their truth is challenged by familiar phenomena. I finish with some remarks that suggest the intellectualist ends up in a better argumentative position.

KEY WORDS: intellectualism, reasons for action, nonconceptual content, phenomenology, absorbed coping

\section{Introducción}

John McDowell es conocido principalmente por la tesis de que el contenido de la experiencia es conceptual. Esto significa que en el caso de animales racionales como nosotros tener experiencias requiere la operación de capacidades que pertenecen a nuestra racionalidad, es decir, aquellas mismas capacidades que usamos para formular juicios (2000). En el proceso de formular y defender esta tesis, McDowell ha defendido una tesis semejante respecto de la acción. Para McDowell, actuar requiere el ejercicio de capacidades racionales. Este ejercicio "le da forma" (informs) a la acción, haciendo de ella la "efectuación" (realization) de un concepto de acción, por ejemplo, del concepto "atrapar esto", "lanzar eficientemente a primera base" o "defender la reina" (al jugar ajedrez). Aunque el conceptualismo de McDowell acerca de la percepción ha sido uno de los principales contrincantes 
en el largo y conocido debate acerca del contenido de la experiencia, su tesis paralela acerca de la acción, tesis que aquí llamaré "intelectualista", ha sido debatida con atención sólo recientemente y a partir de un intercambio con Hubert Dreyfus (Dreyfus 2007a, 2007b, 2007c, 2013; y McDowell 2007a, 2007b y 2013). ${ }^{l}$ Si bien nadie negaría que las acciones fruto de un proceso deliberativo explícito o aquellas en las que monitoreamos reflexivamente lo que hacemos involucran la operación de capacidades racionales, no es evidente para muchos que lo mismo sea cierto de acciones carentes de reflexión o deliberación. El debate con Dreyfus se ha centrado en un conjunto de conductas que han recibido etiquetas tales como "prácticas expertas", "acciones no reflexivas" y "desempeño absorto" (absorbed coping). De manera intuitiva, éstas son conductas con las que respondemos de manera inmediata y habilidosa a las exigencias de una situación sin que medien de forma explícita procesos mentales de reflexión como inferencias, ponderación de alternativas, cálculos prácticos y en general deliberación. Algunos ejemplos notables serían los del violinista virtuoso inmerso en un concierto o la tenista que reacciona magistralmente en medio de las urgencias del partido. Pero estas conductas también abarcan casos omnipresentes de experticia mundana, como la que todos desplegamos usualmente al enfrentarnos a una puerta, al realizar labores domésticas, o en buena parte en nuestra actividad como conductores, peatones, etc. Para McDowell, las conductas de este tipo están "permeadas de conceptualidad", o por decirlo de otro modo, necesariamente involucran el ejercicio de nuestras capacidades conceptuales. Dreyfus, en cambio, considera que debemos entender este tipo de conductas en términos de habilidades y disposiciones en las cuales nuestras capacidades conceptuales no cumplen un papel.

El debate entre McDowell y Dreyfus, que aquí enmarcaré en términos de intelectualismo versus antiintelectualismo, ha llamado la atención sobre ciertos asuntos de interés filosófico a los que tradicionalmente no se les ha considerado lo suficiente, y guarda relaciones potencialmente fructíferas con el debate reactivado recientemente acerca del saber-qué y el saber-cómo, ${ }^{2}$ y tiene implicaciones para la filosofía de la acción en general que van más allá del caso de las

\footnotetext{
${ }^{1}$ Aunque en muchos contextos el término "intelectualismo" se emplea despectivamente, hablaré en estos términos de la posición de McDowell, a pesar de que con ella me alineo. Más adelante aclararé en qué consiste la postura, lo cual justificará la etiqueta. En el bando intelectualista se cuentan, además de McDowell, Gottlieb (2011), Noë (2013), Pippin (2013) y hasta cierto punto Rouse (2013). Con Dreyfus se alinean Carman (2013) y Schear (2013a).

${ }^{2}$ Un artículo de Stanley y Williamson (2001) reinicia el debate, en el que han
} 
acciones no reflexivas o conductas expertas. ${ }^{3}$ Sin embargo, la discusión no ha estado exenta de dificultades respecto de cómo ubicar y especificar el desacuerdo. Tras una etapa de mutua aclaración entre los dos principales protagonistas, en poco tiempo se han ido sumando nuevos participantes cuyas contribuciones, en buena parte, se encuentran en un volumen reciente dedicado al debate (Schear 2013b). En mi opinión, sin embargo, aún no se ha alcanzado el nivel de claridad suficiente para hacer instructivo el debate. En este contexto, mi propósito es doble. Por una parte, argüiré a favor de cierta formulación del desacuerdo. Por otra parte, y a la luz de lo anterior, argumentaré que el principal argumento antiintelectualista es insatisfactorio y que ello deja en una posición de superioridad argumentativa a su contrincante.

Empezaré por explicar cada una de las posiciones, lo que permitirá, entre otras cosas, especificar a qué dominio de conductas aplica la tesis en disputa (secciones 2 y 3 ). A continuación criticaré cierta propuesta acerca de cómo entender el debate, y propondré una alternativa (sección 4). Luego presentaré el principal argumento antiintelectualista y mostraré que es insuficiente debido a que tiene presupuestos para los que no se ha ofrecido motivación y que no son autoevidentes, especialmente a la luz de ciertos puntos concedidos por el antiintelectualista mismo (sección 5). Por último evaluaré la situación dialéctica resultante, y haré consideraciones que aumentan la presión sobre el antiintelectualista (sección 6).

\section{Intelectualismo}

El acuerdo básico del que parte la discusión entre intelectualistas y antiintelectualistas es que en las conductas expertas no reflexivas o de desempeño absorto el agente está "inmerso" en la acción en el sentido de que no ejerce la capacidad de contemplar su propia conducta para hacerla objeto de reflexión, por ejemplo, para monitorearla (Dreyfus 2013; McDowell 2013). En este sentido, Dreyfus y McDowell están de acuerdo en que las conductas cuya naturaleza es el objeto en discusión son no reflexivas. La de McDowell es una posición intelectualista en la medida en que afirma que las capacidades que son esenciales

participado, entre otros, Noë (2005), Bengson y Moffett (2007), Bengson, Moffett y Wright (2009), Fridland (2011) y Stanley (2011).

${ }^{3}$ Inicialmente hablaré indistintamente de conductas expertas, acciones no reflexivas y desempeño absorto, pues así se ha hecho durante el debate. Más adelante identificaré las acciones no reflexivas como aquellas a las que propiamente se aplican las tesis en disputa. 
para el desarrollo de operaciones evidentemente intelectuales, por ser reflexivas, como juzgar, inferir y deliberar, son también esenciales para el desarrollo de las operaciones no evidentemente intelectuales, por ser no reflexivas, involucradas en algunas conductas expertas. Como McDowell (2007a) lo indica, su tesis depende de rechazar la idea de que el único contexto de operación de estas capacidades es el razonamiento, entendiéndose como una operación en algún sentido explícita para el sujeto. ${ }^{4} \mathrm{El}$ debate se puede entender como emanando de un desacuerdo sobre este punto. Sin embargo, yo analizaré la discrepancia en términos distintos.

Tal como se puede recoger de la discusión, el hecho de que las capacidades racionales "informen" la acción no reflexiva consiste, para McDowell, en que la acción posee tres rasgos:

1. Es realizada por razones. Llamaré a esto el hecho de que sea racionalizada. ${ }^{5}$

2. Es individuada. El agente la comprende como siendo cierto tipo de acción. ${ }^{6}$

3. Es entendida por quien la realiza como fruto de su agencia. Es de propiedad de un agente. ${ }^{7}$

A la tesis de que la acción no reflexiva tiene estos tres rasgos la llamaré tesis de la articulación racional de la acción. El debate entre

${ }^{4}$ McDowell no usa mi calificación "entendida como una operación en algún sentido explícita para el sujeto". Que haya razonamientos implícitos, que haya grados de explicitez en el razonamiento, o que haya operaciones racionales que no sean propiamente razonamientos — que es de hecho la posición de McDowell— son puntos que, aunque considero abiertos, dejo de lado. Uso la noción de razonamiento en algún sentido explícito como neutral respecto de las posibilidades mencionadas y confío en que sea intuitivamente clara hasta donde lo requiere el argumento de este escrito.

5 "El autoconocimiento distintivo de un agente no sólo es conocimiento de qué está haciendo sino también de por qué lo está haciendo" (McDowell 2013, p. 47, la traducción es mía). En adelante todas las traducciones son mías.

${ }^{6}$ La idea es que el agente entiende y realiza la acción, por ejemplo, como "subir las escaleras" en lugar de en términos de aquellas otras cosas que se hacen para poder subir las escaleras, como flexionar las piernas así y asá (véase McDowell 2009b, pp. 367-368). Para otro modo de exponer los dos rasgos anteriores véase Gottlieb 2011.

7 "La autoconciencia en la acción es práctica, no teorética. Es una cuestión de 'yo actúo' en lugar de 'yo pienso' [...]. Concebir la acción en términos del 'yo actúo' es una forma de registrar el carácter de primera persona que tiene, de manera esencial, la efectuación de capacidades racionales prácticas, en las cuales consiste actuar" (McDowell 2007a, p. 367). 
intelectualistas y antiintelectualistas, en mi formulación, es acerca de la verdad de esta tesis. Que una acción posea estos rasgos no necesariamente implica - $-\mathrm{y}$ en el caso de las conductas no reflexivas no consiste en - que los rasgos sean el contenido de ciertos pensamientos del agente al momento de realizar la acción; es decir, el agente no necesariamente debe tener pensamientos con contenidos tipo "esto es una razón para hacer esto", "yo actúo", ${ }^{8}$ o "estoy subiendo las escaleras". 9 La idea de McDowell es que el agente puede saber estas cosas sin necesidad de formularse mentalmente los pensamientos correspondientes.

En la etapa inicial del debate parecía que Dreyfus y McDowell agrupaban del mismo modo las acciones a las que se aplican sus respectivas tesis. Sin embargo, en la última contribución de McDowell (2013) quedó claro que no es así. Hay un subconjunto de conductas que Dreyfus considera expertas y a las que McDowell no pretende aplicar la tesis intelectualista. El ejemplo que se discute es el de los movimientos que realizamos al tratar de mantener una distancia socialmente aceptable de otras personas según el contexto (una conversación, un ascensor, etc.). ${ }^{10}$ El criterio para establecer cuáles conductas son articuladas racionalmente es que, respecto de estas conductas, sea procedente dirigir la pregunta "¿por qué?" al agente. Evidentemente, si la pregunta se formula mientras el agente está inmerso en la acción, tendrá que interrumpir la inmersión y pasar a un estado reflexivo, pero lo importante es que la acción sobre la que se pregunte haya sido realizada de un modo no reflexivo. McDowell no ofrece una especificación de este criterio; sin embargo, hace explícita su deuda con Anscombe (1963), quien sí trató de precisar cuál sentido de la pregunta "¿por qué?" era el relevante, aunque su objetivo era identificar las acciones intencionales. Creo que es posible evitar adentrarnos en los pormenores del análisis de Anscombe diciendo que el sentido relevante de la pregunta “¿por qué?” para el debate sobre la acción no reflexiva es aquel en que las razones que se piden son razones para el agente, esto es, estados que el agente reconoce como motivaciones racionales de su acción y que, por lo tanto, puede ver como entrando en relaciones de justificar, descartar, o en

${ }^{8}$ La afirmación expresa que no es necesario un pensamiento con el contenido "yo actúo". Pero McDowell propone que un "yo actúo" es algo así como la forma de la acción (véase la nota anterior).

${ }^{9}$ Suponiendo que "estoy subiendo las escaleras" sea el tipo de contenido de una intención en acción, como arguye McDowell (2010).

${ }^{10}$ La tesis intelectualista típicamente no aplica para este tipo de conducta, lo cual no significa que no aplique en ningún caso. 
general hablar a favor o en contra de un curso de acción. Aunque hay que admitir que esta noción de razones para el agente está lejos de ser totalmente clara, confío en que para la discusión que sigue será suficiente la formulación dada. En cualquier caso, creo que esta formulación captura una noción general de razones que McDowell y Dreyfus comparten y a partir de la cual se erige su desacuerdo.

Que la pregunta "¿por qué?” sea aplicable significa que el agente la reconoce como procedente, como una pregunta para la cual puede ofrecer una respuesta adecuada. Una indicación clara de que la pregunta no es procedente sería que el agente expresara desconcierto ante la implicación de que ejecutó la acción a la que la pregunta alude. Por ejemplo, en el caso de los movimientos que realizamos para mantener una distancia adecuada de otras personas la pregunta “¿por qué?" muy probablemente se toparía con una respuesta tipo “¿de verdad hice eso?” (McDowell 2013). Por esta razón, este tipo de conductas no deben considerarse racionalmente articuladas. ${ }^{11}$

Podemos ahora precisar la discrepancia entre intelectualistas y antiintelectualistas. Llamaré "acción no reflexiva" a aquellas conductas sobre las que versa el debate. Una acción es no reflexiva si es una conducta experta no reflexiva sobre la cual tiene sentido dirigir al agente la pregunta "¿por qué?", entendida como pidiendo las razones del agente para realizar la acción. El desacuerdo es el siguiente: el intelectualista considera que las acciones no reflexivas son articuladas racionalmente, es decir, que son racionalizadas, de propiedad del agente e individuadas, mientras que el antiintelectualista lo niega. El antiintelectualista cree que el que tenga sentido hacer y responder la pregunta “ipor qué?", respecto de estas acciones, no significa que la acción sobre la que se pregunta haya sido articulada al momento de su realización. Esta capacidad de dar cuenta de la acción puede entenderse simplemente como una racionalización post hoc (Rietveld 2010; Noë 2013). Hablaré sobre esto un poco al final de este trabajo. De momento, es importante apreciar que a pesar de la acotación del debate el desacuerdo sigue siendo bastante significativo, pues hay un grupo muy amplio de conductas expertas no reflexivas para las que el antiintelectualista admite la pertinencia de la pregunta "¿por qué?" aunque niega que estén articuladas racionalmente. Baste decir que con excepción del caso de la distancia social todos los ejemplos

11 "Si alguien está haciendo algo que no sabe que está haciendo, como sin duda puede ocurrir, su acción [her doing] queda fuera del dominio de la tesis de la ubicuidad en su aplicación a la acción" (McDowell 2013, p. 50). La tesis de la ubicuidad de lo mental (pervasiveness of the mental) es la que yo he especificado, para la acción, en términos de articulación racional. 
mencionados hasta el momento en este artículo pertenecen a este grupo.

La motivación principal para la tesis de la articulación racional de la acción no reflexiva se sugiere en el pasaje en el que McDowell discute la diferencia entre que un perro atrape un frisbee y que lo haga una persona:

El sentido de decir que, a diferencia del perro, la agente racional está efectuando un concepto al hacer lo que está haciendo es que, bajo una especificación que capture el contenido del concepto práctico que ella está efectuando, su acción cae en el ámbito de su racionalidad práctica -incluso si esto ocurre sólo en la medida en que si se le preguntara por qué atrapó el frisbee ella contestara "por ninguna razón en particular, simplemente quise hacerlo"-. (McDowell 2007b, p. 369)

Que una acción caiga en el ámbito de la racionalidad práctica de un agente significa, en mi interpretación, que para el agente la acción tiene los tres rasgos mencionados anteriormente (racionalización, individuación, propiedad). El caso que contempla McDowell en este pasaje es justamente el caso límite en que la agente puede responder la pregunta por las razones sólo de un modo extremadamente indeterminado - lo cual, sin embargo, no significa que la acción no sea racionalmente articulada-. Pero es fácil concebir casos que parecen más dicientes. Un jugador de fútbol que en medio de las urgencias del juego lanza un pase a mengano en vez de a fulano, puede explicar su acción retrospectivamente citando razones, algunas incluso bien desarrolladas como "si le hubiera lanzando el pase a fulano, éste habría quedado en fuera de lugar". No parece improbable que el jugador reconozca que no reflexionó sobre qué hacer cuando se vio en la necesidad de lanzar el pase, esto es, que no intervino ningún razonamiento práctico en su acción. Y sin embargo esto no impediría al jugador reconocer la acción como fruto de su agencia y como realizada por razones. Un punto adicional que le da fuerza a esta forma de ver la acción no reflexiva, es que en muchos casos, aquel a quien se le pregunta por su acción, como el jugador de fútbol, puede dar cuenta de ella de forma inmediata, esto es, sin detenerse a reflexionar sobre ella (McDowell 2013). Esta capacidad para explicar la acción no reflexiva propia en términos que la retratan como racionalmente articulada, sin que para hacerlo parezca hacer falta un proceso adicional de reflexión retrospectiva sobre la acción es un hecho que parece innegable y, por lo tanto, que el antiintelectualista debe poder explicar. Ya volveremos sobre esto. En cualquier caso, 
para McDowell todo esto sugiere que el agente inmerso en la acción no reflexiva "sabe lo que está haciendo y por qué" (McDowell 2013, p. 48).

\section{Antiintelectualismo}

Por su parte, Dreyfus defiende que la acción no reflexiva debe entenderse como el fruto de conjuntos de disposiciones y habilidades en cuya actualización y ejercicio no participa nuestra racionalidad. Dreyfus vincula este tipo de acción con el estado de saber-cómo, entendido, evidentemente, de un modo no intelectualista. ${ }^{12} \mathrm{Si}$ bien Dreyfus admite que durante el proceso de aprendizaje, de adquisición de habilidades y disposiciones, las capacidades conceptuales pueden (aunque no necesariamente deben) desempeñar un papel, lo importante es que dejan de ser relevantes una vez que estas habilidades y disposiciones han sido asimiladas:

Una vez adquirimos una habilidad, los conceptos empleados al aprender dicha habilidad no tienen que desempeñar ningún papel adicional. $\mathrm{Ni}$ siquiera es necesario que al aprender una práctica se necesite ser consciente de los conceptos relevantes. Nuestra habilidad para actuar normalmente es adquirida al imitar autoridades sin que los conceptos desempeñen ningún papel consciente. $(2013$, p. 18)

Uno de los instrumentos que Dreyfus emplea para explicar su posición es la noción de intencionalidad motora que Merleau-Ponty desarrolla. Pónganse el caso de tomar una taza de café mientras se está distraído leyendo algo. La capacidad para hacer esto es un caso de experticia que muchos compartimos. Evidentemente, la mayor parte de las veces en que realizamos este tipo de acción no hay intermediación explícita de un proceso de razonamiento o deliberación. Pero hay algo más que hace interesantes estos casos. Cuando la acción de tomar la taza de café se inicia, el movimiento del brazo y la forma que adopta la mano parecen tomar en cuenta, de manera muy específica, un buen número de factores como la forma de la taza, su tamaño, fragilidad, orientación, peso, contenidos, etc. Esta especificidad del componente puramente motor queda fuera de la atención del agente, al tiempo que sugiere que la acción desde el comienzo está dirigida a una meta, y por ende tiene condiciones de éxito. Pero además,

${ }^{12}$ No intelectualista en el sentido propio del debate sobre saber-cómo, no en el que discutimos en este artículo. El antiintelectualista en aquel debate es quien defiende que el saber-cómo no es una especie del saber-qué (véase la nota 2). 
es este componente motor el que hace que la acción tenga éxito, a pesar, por ejemplo, de realizarse a menudo con relativamente poca retroalimentación visual (Kelly 2000). ${ }^{13}$

La acción experta no reflexiva es, pues, gobernada por un tipo de normatividad distinto al de las acciones en las que opera nuestra racionalidad. Aunque hay condiciones de éxito (por ejemplo, lograr un pase eficiente en fútbol), lo cual hace que esta acción no pueda concebirse a la manera de un acto reflejo, estas condiciones no están presentes para el agente de un modo compatible con la tesis de la articulación racional de la acción. Para ver esto es importante precisar el sentido en el que las acciones no reflexivas tienen propósito y motivación en la concepción dreyfusiana. En sentido estricto, se adscribe propósito a la acción no reflexiva en virtud de los objetivos trazados para ese tipo de acción en el pasado, particularmente en el periodo de aprendizaje, cuando dichas acciones no eran realizadas de forma no reflexiva. Si todo sale bien, la acción experta no reflexiva en cierto sentido alcanza estos objetivos, pero esto ocurre "sin que el agente, consciente o inconscientemente, tenga que tenerlos en mente como metas" (Dreyfus y Dreyfus 1999, p. 113). Puesto así, es claro que éstos son propósitos de la acción sólo en un sentido derivativo, pues propiamente no forman parte de la motivación de la acción. La motivación como tal consiste en la tendencia a alcanzar lo que Dreyfus, siguiendo a Merleau-Ponty, llama el "agarre máximo" (maximum grip) de la situación, es decir, la tendencia a reducir lo que, en virtud de nuestra experticia, experimentamos como desequilibrio o tensión al realizar la acción. Esto es lo que permite mantenernos inmersos en el flujo de la situación (Dreyfus y Dreyfus 1999). Lo importante es que esta presencia de propósitos y motivaciones no requiere el ejercicio de capacidades conceptuales, sino sólo las habilidades y disposiciones necesarias para responder a las exigencias cambiantes de la situación de una manera adecuada, donde "adecuado" remite, por una parte, a las metas explícitas durante el proceso de aprendizaje $-\mathrm{y}$ en rigor no presentes en el momento de la acción experta-, y por otro, a cierta sensación de equilibrio o de fluir en la acción.

\footnotetext{
${ }^{13}$ Kelly contrasta esta acción de agarrar con la de señalar e incluso con la de sólo tocar, las cuales requieren gran cantidad de información visual y una identificación del objeto en un espacio objetivo, identificación que no parece ocurrir para el caso de agarrar.
} 


\section{4. ¿Un falso debate?}

Joseph Rouse (2013) ha sugerido que el desacuerdo entre McDowell y Dreyfus es en buena medida insustancial dado que las posiciones que defienden responden a problemas diferentes y son potencialmente compatibles. Creo que la propuesta de Rouse es errada, pero apreciar por qué lo es puede ser instructivo para precisar el tema del debate. Para justificar su propuesta, Rouse distingue entre enfoques descriptivos y enfoques normativos del contenido conceptual. Los enfoques descriptivos "toman el contenido conceptual como algo que está de hecho presente o es operativo en ciertas actuaciones específicas de los usuarios de conceptos" (2013, p. 250). Los enfoques normativos, en cambio, "identifican el dominio conceptual con aquellas actuaciones y capacidades que son evaluadas apropiadamente en términos de normas racionales" (p. 250), "la cuestión es si la evaluación en términos de normas conceptuales es apropiada" (p. 251), no si durante la actuación en cuestión ocurrieron de hecho operaciones racionales. La sugerencia de Rouse es que las críticas de Dreyfus sólo aplican al enfoque descriptivo, mientras que la propuesta de McDowell es estrictamente normativa.

En principio, la sugerencia de Rouse parece encajar bien con cierto aspecto del espíritu de cada uno de los filósofos en cuestión. ${ }^{14}$ No obstante, creo que la interpretación de Rouse hace muy modesta la posición intelectualista de McDowell. Si bien hablar de "hacer que la acción quede dentro del ámbito de la racionalidad práctica de la agente", por parafrasear a McDowell, puede sugerir que el problema es simplemente interpretativo, esto es, una cuestión de revisar qué tan apta es una acción para ser puesta en términos normativo-racionales, es claro que a McDowell le interesa principalmente qué pasa con la acción en el momento de su realización, no sólo cómo se interpreta retrospectivamente. ${ }^{15}$ En un sentido muy específico la posición de

${ }^{14}$ En buena medida la carrera de Dreyfus se ha cimentado sobre sus críticas a proyectos descriptivos de la cognición. Su obra clásica es What Computers Can't Do (1972). Por su parte, McDowell ha rechazado en varias oportunidades los proyectos metafísicos explicativos, y ha defendido que el tipo de inteligibilidad que otorga considerar cierta conducta como articulada de acuerdo con normas racionales es irreductible a, e irremplazable por, la que otorgan las ciencias naturales (2000) o las ciencias cognitivas (1994).

${ }^{15} \mathrm{Al}$ criticar uno de los argumentos de Dreyfus por errar el blanco, un argumento que apela al ejemplo de los maestros de ajedrez y de la agente que atrapa un frisbee, McDowell afirma: "Para rechazar la tesis de la ubicuidad de lo mental, lo que él debería argüir es que las capacidades conceptuales no son operativas en la acción del maestro de ajedrez o de quien atrapa un frisbee al actuar como lo hacen. 
McDowell también es descriptiva: dice qué ocurre en la realización de la acción. ${ }^{16}$ Pero el sentido es específico porque McDowell no concibe su posición a la manera de los casos que Rouse usa para ilustrar el enfoque descriptivo, como el trabajo de Fodor o de los usuales oponentes de Dreyfus, normalmente matriculados en el proyecto de las ciencias cognitivas y que, de aceptar cierto reductivismo, McDowell caracterizaría como naturalistas en un sentido no aprobatorio. ${ }^{17}$

¿Cómo situar entonces la posición de McDowell? Mi propuesta es interpretar la tesis intelectualista como una respuesta a la pregunta "¿cómo es la acción para la agente al momento de su realización?". De acuerdo con esto, la respuesta es que la acción es articulada racionalmente en el sentido especificado. ${ }^{18}$ Este modo de entender la posición intelectualista excluye tanto un enfoque meramente normativo, compatible con la idea de que la acción se presente al agente como racionalmente articulada sólo en la medida en que es evaluada, como un enfoque descriptivo de corte naturalista en que se trata de evitar tomar una perspectiva normativa-racional como punto de partida irreductible. Una virtud de la reformulación que he propuesto de la pregunta básica, a saber, “¿cómo es la acción no reflexiva para el agente al momento de su realización?", es que no sólo no excluye la respuesta antiintelectualista de Dreyfus, sino que se aviene bien con su enfoque fenomenológico. Esto significa que la argumentación que sigue a favor de la posición de McDowell no incurre en circularidad; o al menos no en una que emane de mi formulación del problema básico.

\section{El argumento fenomenológico del contenido sin objetos}

Tras los ires y venires del debate entre Dreyfus y McDowell, parece claro que el argumento más significativo del primero en contra de la tesis del segundo es el que Schear (2013a) ha llamado el argumento

(Sin duda es evidente que las capacidades conceptuales sí son operativas en sus respuestas significativas cuando se les piden razones)" (2013, p. 50; las cursivas son de McDowell).

${ }^{16}$ Este punto es equivalente a la posición de McDowell con respecto a la experiencia: no se trata de si la experiencia puede conceptualizarse retrospectivamente, más bien si en la misma adquisición de experiencias están en juego capacidades conceptuales.

${ }^{17}$ Lo que él llama "naturalismo crudo" (McDowell 2000, conferencia 4). El sentido es no aprobatorio por lo mencionado en la nota 14 .

${ }^{18}$ Esto es consistente con la siguiente afirmación: "El autoconocimiento que el agente expresa [en respuesta a la pregunta “¿por qué?'] no empieza a existir al momento de ser expresado" (McDowell 2013, p. 46). 
fenomenológico de la fusión, ${ }^{19}$ y que yo llamaré del "contenido sin objetos". Se puede poner de la siguiente manera: ${ }^{20}$

P1. El contenido de los estados que son objeto de la operación de capacidades conceptuales debe ser especificado en términos de una ontología de objetos.

P2. El contenido involucrado en la acción no reflexiva no puede ser especificado adecuadamente en términos de una ontología de objetos. $^{21}$

C. Luego, el contenido involucrado en la acción no reflexiva no es objeto de la operación de capacidades conceptuales.

Para nuestros propósitos basta con la siguiente explicación general de (P1): las capacidades racionales son capacidades empleadas para formar juicios; por ende, involucran capacidades conceptuales. La concepción tradicional de contenido conceptual en juego en (P1) está inspirada en Frege y en ella "el contenido conceptual presenta el mundo al sujeto como divido en objetos, propiedades y situaciones: los componentes de las condiciones de verdad" (Cussins 2002, p. 134). La idea es, por lo tanto, que de admitirse que las capacidades conceptuales están involucradas en la acción no reflexiva, debemos aceptar que el contenido que proporcionan responde a una ontología de objetos, propiedades y situaciones. No pondré en duda esta premisa, entre otras cosas, porque no parece ser un punto que McDowell rechazaría. ${ }^{22}$

${ }^{19}$ Schear considera y descarta otro argumento llamado "del error categorial". En mi opinión el argumento está claramente desencaminado, y no por las razones que aduce Schear. Aquí resulta necesario dejarlo de lado. Gottlieb (2011) considera y descarta lo que llama "el argumento de la velocidad", que en mi opinión tampoco tiene mucha fuerza.

${ }^{20}$ He hecho algunos cambios respecto de la forma en que Schear (2013a, p. 294) presenta el argumento. Por una parte, he especificado la formulación de Schear en términos de contenido para evitar alguna ambigüedad. Por otra, he omitido un paso adicional del razonamiento que responde al hecho de que Schear quiere llegar a una conclusión ulterior que aquí no nos interesa.

${ }^{21}$ Aunque Dreyfus y McDowell discuten a este respecto principalmente el contenido de la percepción, dando por supuesto que la percepción tiene contenido - cosa que no todo filósofo admite (véase Travis 2004, 2007) - , se debe tener presente que McDowell (2010) considera en una discusión cercana otro contenido, el de la intención en acción. Este contenido tendría que ser admitido por McDowell para la acción no reflexiva, pues justamente es el que especifica la descripción bajo la cual el sujeto entiende su acción, lo cual, como se verá es fundamental en mi comprensión del problema.

${ }^{22}$ Para una mejor forma de dar contenido a esta premisa en un contexto semejante, véase Cussins 1992. 
Lo importante y potencialmente controversial radica en (P2). Una de las descripciones fenomenológicas aducidas por Dreyfus para motivar esta premisa proviene de Merleau-Ponty:

Para el jugador en acción el campo de fútbol no es un "objeto". Está atravesado por líneas de fuerza (las líneas que marcan el área de penalti) y está articulado en sectores (por ejemplo las "aperturas" entre adversarios), que exigen ciertos modos de acción. El campo mismo no es dado [al jugador], sino que le está presente como el término inmanente de sus intenciones prácticas. El jugador se siente uno con éste y siente la dirección de la portería, por ejemplo, tan inmediatamente como los planos horizontales y verticales de su propio cuerpo $[\ldots]$ En ese momento la conciencia no es nada excepto la dialéctica de medio y acción. Cada maniobra que el jugador realiza modifica el carácter del campo y establece nuevas líneas de fuerza en las que a su vez se desenvuelve y se realiza, alterando una vez más el campo fenoménico. (Merleau-Ponty 1965, pp. 168-169, citado en Dreyfus 2007c, p. 106; las cursivas son de Dreyfus)

La idea que toma Dreyfus de Merleau-Ponty es que la descripción fenomenológica de la acción no reflexiva del jugador de fútbol no remite a un contenido especificable en términos de objetos y sus propiedades, y de un sujeto separado de éstos. En otros pasajes, Dreyfus afirma, usando un término de psicología de la Gestalt, que la experiencia de quien actúa no lo es de objetos sino de solicitaciones (solicitations), las cuales se caracterizan por el hecho de no ser percibidas como algo allá afuera en relación con el cual el sujeto decide actuar, sino que percibirlas es sentirse inmediatamente atraído hacia (o repelido por) ellas (2007c, p. 104, nota 1). Por esta razón, Dreyfus hace eco de pasajes en los que Merleau-Ponty se permite hablar incluso de que en este tipo de acciones no hay experiencia de un yo separado sino que yo me "fundo" con mi cuerpo, a la vez que mi cuerpo no es algo separado de la red de solicitaciones $(2007 \mathrm{c}$, p. 356).

Creo que el argumento fenomenológico del contenido sin objetos no es exitoso. Una primera inquietud acerca de esta posición es qué tan apropiado es el testimonio fenomenológico como instrumento argumentativo en este contexto, tanto por principio, como debido a ciertas anomalías del testimonio de expertos (Gottlieb 2011). A esto se puede añadir que el testimonio de expertos a veces parece desafiar las descripciones fenomenológicas de Dreyfus (Montero 2013), sin contar que en muchos casos varía ostensiblemente. No quiero, sin embargo, 
simplemente descartar que la fenomenología cumpla un papel en el examen de problemas como el que está en discusión, entre otras cosas, porque McDowell mismo no lo hace (2007a, p. 349). Tomemos entonces el caso de la descripción fenomenológica de cómo son las cosas para el jugador durante (al menos en ciertos momentos de) el partido de fútbol. La idea merleau-pontiana es que el contenido involucrado para el jugador es capturado por conceptos como "líneas de fuerza", "aperturas", y conceptos de un espacio relativo al cuerpo del jugador y por lo tanto constantemente cambiante (dado como se desarrolla la situación). Como primer paso para afirmar (P2), el fenomenólogo antiintelectualista debe seguir el esquema no conceptualista y sostener que estos conceptos, sin embargo, son herramientas teóricas para dar cuenta de un contenido que para el agente es no conceptual; esto significa que el agente no necesita tener los conceptos en cuestión para poder tener el contenido que estos conceptos capturan. (P2), por lo tanto, debe implicar que el contenido involucrado en la acción no reflexiva es no conceptual. Sin duda sería ridículo suponer que el jugador de fútbol necesita un concepto como "línea de fuerza" para poder captar ciertas oportunidades y jugadas posibles. Pero la negación no es suficiente para soportar (P2). Para ver esto tomemos un ejemplo de solicitación discutido por Dreyfus y McDowell, a saber, el de una apertura percibida como del tamaño apropiado para el cuerpo propio en un momento en que es necesario pasar al otro lado de una pared con urgencia. Incluso si uno otorga al fenomenólogo antiintelectualista que fenomenológicamente la percepción de la apertura en cierto sentido no es separable de la "atracción" que genera (y es esto lo que permite hablar de "solicitación", como vimos), resulta difícil pensar en esta inseparabilidad como algo que impida hablar de la percepción de un objeto (una apertura) poseedor de ciertas propiedades, así sean relacionales (ser lo suficientemente grande para que pase mi cuerpo). Más aún, tampoco parece haber ningún problema, como McDowell plantea (2007a), en decir que el hecho de que veamos que la apertura tiene el tamaño apropiado es una razón que nos motiva de forma inmediata a atravesar la pared por ese espacio. Ésta es la idea que quiero desarrollar, la cual entiendo así: la descripción fenomenológica del contenido sin objetos es compatible con una descripción adecuada de cómo son las cosas para el agente que revele la articulación racional de la acción. Una solicitación puede ser una razón.

Es importante empezar por anotar que mi propuesta es compatible con admitir que conceptos como el de "solicitación", u otros más 
específicos como el de "línea de fuerza", capturan aspectos no conceptuales de cómo son las cosas para el agente. En otras palabras, mi propuesta es neutral respecto de si hay o no contenido no conceptual; sólo afirma que si lo hubiera, esto no descartaría que haya también el tipo de contenido que requiere la postura intelectualista para ser verdadera. Esto es una ventaja sobre la posición de McDowell mismo, quien tanto para la acción como para la percepción rechaza el contenido no conceptual provocando varias objeciones (por ejemplo, para la percepción, véase Peacocke 1998, Kelly 2001, Travis 2004, 2007). Mi versión, más modesta, permite el avance del punto intelectualista con respecto a la acción sin depender de responder a las protestas no conceptualistas.

La idea clave de la que parte mi propuesta es conocida en la filosofía de la acción: lo que una persona hace admite varios niveles de descripción. Estos niveles de descripción presentan los haceres del agente de diversas formas: como procesos subpersonales, como causas de eventos de los cuales el agente es ignorante, o como acciones intencionales en un sentido no (o menos) problemático. ${ }^{23}$ Esta variedad de descripciones da pie al problema de la individuación de la acción. Sin embargo, la sugerencia que quiero hacer no requiere una solución a esta cuestión. Mi sugerencia es que la tesis intelectualista se puede entender así:

T: Para toda acción no reflexiva $N$ de un agente $A$, hay una descripción de $N$ en términos de un concepto de acción $\emptyset$, tal que $A$ sabe 1) que está $\emptyset$-ando, y 2) por qué está $\emptyset$-ando.

Esta formulación es una negación de (P2), pues el saber mencionado en (T) involucra, evidentemente, contenido conceptual. Sin embargo, como he anticipado, la descripción fenomenológica antiintelectualista no justifica (P2), pues incluso si aceptáramos i) que este tipo de descripción captura cómo son las cosas para el sujeto absorto en la acción no reflexiva, y ii) que el contenido capturado es no conceptual para el agente, esto no descarta que haya otra descripción de la acción no reflexiva que además de capturar cómo son las cosas para el sujeto, presente la acción como racionalmente articulada, esto es, no descarta (T). Para descartar (T) y por ende sacar a flote (P2), habría que añadir a (i) y (ii), una de las siguientes tesis:

${ }^{23}$ Esta observación no pretende ofrecer una clasificación tripartita de los modos de descripción de lo que hace un agente, sólo ilustrar, de forma muy general, tres tipos de casos entre otros posibles. Cabe anotar, también, que cada uno de estos tipos de casos admiten varias descripciones. Para discusiones clásicas de este punto véanse Anscombe 1963 y Davidson 1963. 
a) Quien realiza una acción no reflexiva no sabe lo que hace.

b) Quien realiza una acción no reflexiva no sabe lo que hace bajo una descripción sino de otro modo.

Unida a (i) y (ii), (a) implica que la relación de quien realiza la acción no reflexiva con esta acción no es de saber, lo cual descarta a (T), dejando a (P2) en principio intacta, pues el antiintelectualista podría tratar de argüir que se puede tener una relación con contenido no conceptual sin que esta relación sea de saber. (b), por su parte, deja abierta la posibilidad de que la relación de quien realiza la acción no reflexiva con ésta sea de saber, pero descarta que sea el tipo de saber conceptual involucrado en (T).

Es difícil ver cómo (a) podría ser correcta para todas las acciones no reflexivas sobre las que intelectualistas y antiintelectualistas discuten. Sin duda, hay ocasiones en que hablamos de haber actuado como autómatas refiriéndonos a que no sabemos exactamente cómo terminamos en cierta situación a pesar de que llegar a ella tuvo que involucrar la realización de tareas complejas de nuestra parte. Piénsese en lo rutinario que puede llegar a ser conducir hasta el lugar de trabajo, y el hecho de que por lo mismo uno puede no recordar los detalles de un recorrido específico. Sin embargo, no resulta fácil darle sentido a la tesis de que mientras conducíamos al trabajo no sabíamos que estábamos conduciendo. La dificultad aumenta cuando se trata de acciones no reflexivas expertas pero no exactamente rutinarias, como la del pianista inmerso en su actuación. Esto nos lleva a discutir (b). Esta tesis afirma, por una parte, que en la acción no reflexiva hay un modo de conocimiento no conceptual sobre lo que se está haciendo, por otra parte, afirma que no hay ningún otro modo de conocimiento. Es importante insistir en que el primer punto por sí sólo es inocuo para el intelectualista; sólo en conjunción con el segundo tenemos un desacuerdo relevante. Sin duda alguna, se han desarrollado posiciones afines al antiintelectualismo que proponen formas de conocimiento y experticia que no son conceptuales. Por ejemplo, es común apelar a la noción de un trasfondo preconceptual que posibilita nuestro desempeño inteligente (Hutto 2012 y Cappuccio y Wheeler 2012) o apelar a la noción de saber-cómo, bajo el supuesto de que este tipo de conocimiento no es una especie de saber-qué (Dreyfus 2007b). Sin embargo, mi punto no es que el anti-intelectualista carezca de una teoría plausible de este tipo de saber o experticia, sino que no ha descartado que también haya operaciones racionales de otro nivel involucradas en la acción no reflexiva. Por ejemplo, es perfectamente posible aceptar que la acción no reflexiva involucra saber-cómo 
(no proposicional), a la vez que se afirma que también involucra otro saber, un saber-qué. Simplemente habría que reconocer que este saber-qué no reduce el saber-cómo en juego, sino que versa sobre el tipo de acción que se está realizando y las razones que tenemos para realizarla. En otras palabras, es el saber-qué al que se alude en (T). Piénsese en el ejemplo clásico del saber montar en bicicleta. Independientemente de que este saber sea irreductible a un saber proposicional, sería ciertamente implausible afirmar de alguien que está montando en bicicleta que no sabe que está montando en bicicleta. Lo que el antiintelectualista no ha producido es un argumento que descarte esta posibilidad a pesar de su plausibilidad inicial.

\section{La racionalización post hoc}

En el supuesto de que estoy en lo cierto con respecto a las limitaciones del argumento del contenido sin objetos, ¿cómo quedan las cosas para los dos contrincantes? Un análisis completo de esto supera el propósito de este trabajo, sin embargo, me permito algunas sugerencias. El antiintelectualista nos debe un argumento para (b). Pero más allá de eso, podemos, creo, imprimir algo más de presión a su tarea. Ya mencioné que con frecuencia parecemos ser capaces de dar cuenta - de forma retrospectiva - de nuestra acción no reflexiva en términos de qué estábamos haciendo y qué razones teníamos para ello. Esto, con frecuencia, lo hacemos sin que parezca necesario que reflexionemos mucho sobre la acción: en muchas ocasiones, a la pregunta de por lo que hicimos y nuestras razones, podemos responder de manera casi inmediata. Como mencioné, el antiintelectualista tiene una forma de dar cuenta de este fenómeno, a saber, diciendo que se trata de la racionalización post hoc de una acción que al ser ejecutada no era articulada racionalmente. En este sentido, el antiintelectualista hace de la práctica de dar cuenta racionalmente de las acciones no reflexivas, una práctica putativa, una que pasa por ser algo que en realidad no es. Hay varias otras prácticas asociadas que también se hacen putativas bajo la explicación antiintelectualista, como la de buscar por qué razón hicimos algo (y a veces descubrirlo), evaluar qué tan apropiadas fueron nuestras razones para hacer lo que hicimos y mentir sobre nuestras razones.

¿Qué tan buena es la explicación antiintelectualista de estas prácticas putativas en relación con la intelectualista? El intelectualista propone que el agente puede dar cuenta racional de su acción no reflexiva, con frecuencia de forma inmediata, porque la acción ya estaba articulada racionalmente, de modo que el agente ya sabía lo que 
hacía y por qué. El ejercicio de algunas de las capacidades que hacen posible la acción (específicamente las capacidades conceptuales involucradas) es el mismo que hace posible dar cuenta de la acción. En cierto sentido, se puede decir, no es necesario postular esfuerzos adicionales por parte del agente/sujeto. En contraste, el antiintelectualista propone que las capacidades involucradas en la realización de la acción son diferentes (por ser no conceptuales) de las involucradas en dar cuenta de la misma (evidentemente conceptuales). El segundo conjunto de capacidades empieza a operar sólo después de realizada la acción y si hay necesidad de dar cuenta de ella - por ejemplo, porque alguien pide razones al agente-. Esta explicación, por lo tanto, atribuye un esfuerzo adicional al agente/sujeto, un esfuerzo que, es importante notar, es particularmente alto en los casos en que el agente da cuenta de su acción de forma inmediata. La razón es la siguiente. De acuerdo con la idea de la racionalización post hoc ésta siempre debe tomar un fenómeno pasado y formalmente extrínseco a la capacidad, por ser no conceptual, y darle una forma conceptual frecuentemente sofisticada, y además aceptable o plausible para otras personas (para empezar, para quien pide una explicación al agente). En los casos en los que el agente da cuenta de su acción de forma inmediata, todo este proceso debe ocurrir de manera casi automática. La exigencia es aun mayor cuando se trata de prácticas como mentir sobre nuestras razones (para acciones no reflexivas), pues el ejercicio de racionalización retrospectivo involucra, al menos, y de manera muy general, los siguientes pasos:

1. Una racionalización retrospectiva de nuestra acción que dé cuenta de nuestra acción.

2. La formación de un juicio de que no es conveniente, apropiado, etc., trasmitir al interlocutor el producto de dicha racionalización.

3. Una nueva racionalización retrospectiva cuyo producto resulte conveniente, apropiado, etc., trasmitir al interlocutor.

Si es verdad, como muchos pensamos, que hay excelentes mentirosos, entonces, desde el punto de vista antiintelectualista, éstos serían capaces de un despliegue muy sofisticado de capacidades conceptuales en lapsos mínimos de tiempo. En otras palabras, la explicación antiintelectualista de nuestra capacidad de racionalizar la acción no reflexiva presupone que sí somos capaces de un ejercicio fluido, sofisticado y al menos casi automático de nuestras capacidades conceptuales. La 
postura antiintelectualista queda abierta, entonces, a dos desafíos. Primero, debe argumentar qué virtudes tiene esta explicación por encima de la mucho más simple del intelectualista. Ligado a esto está, en segundo lugar, que debe explicar qué justifica postular un ejercicio de capacidades racionales tan efectivo, automático, fluido y aparentemente no reflexivo para la racionalización post hoc y negarlo para la acción no reflexiva misma.

\section{Conclusión}

En este artículo he propuesto una manera de especificar el desacuerdo entre las posturas intelectualista y antiintelectualista respecto de las acciones no reflexivas. He ofrecido una interpretación del que parece ser el argumento principal del antiintelectualista, mostrando que, a la luz de consideraciones prima facie plausibles, una de sus premisas requiere justificación ulterior. Termino señalando dificultades adicionales de la postura antiintelectualista con respecto a prácticas comunes relacionadas con dar cuenta racional de nuestras acciones no reflexivas. ${ }^{24}$

\section{BIBLIOGRAFÍA}

Anscombe, G.E.M., 1963, Intention, Harvard University Press, Cambridge, Mass./Londres.

Bengson, J. y M. Moffett, 2007, "Know-How and Concept Possession", Philosophical Studies, vol. 136, no. 1, pp. 31-57.

Bengson, J., M. Moffett, y J. Wright, 2009, "The Folk on Knowing How", Philosophical Studies, vol. 142, no. 3, pp. 387-401.

Cappuccio, M. y M. Wheeler, 2012, "Ground-Level Intelligence: ActionOriented Representation and the Dynamics of the Background", en Radman 2012.

Carman, T., 2013, "Conceptualism and the Scholastic Fallacy", en Schear 2013b.

Cussins, A., 2002, "Content, Conceptual Content and Nonconceptual Content", en Y.H. Gunther (ed.), Essays on Nonconceptual Content, MIT Press, Cambridge, Mass./Londres.

${ }^{24}$ Agradezco a los integrantes del grupo de investigación "Lógica, epistemología y filosofía de la ciencia" y a oyentes de la presentación de una parte de este texto en el III Congreso de la Asociación Latinoamericana de Filosofía Analítica y III Congreso de la Sociedad Brasilera de Filosofía Analítica, así como a dos revisores anónimos por sus excelentes observaciones y sugerencias. Este artículo forma parte de una investigación financiada por la Pontificia Universidad Javeriana, como parte del grupo De Interpretatione. 
Cussins, A., 1992, "Content, Embodiment and Objectivity: A Theory of Cognitive Trails", Mind, vol. 101, no. 404, pp. 652-688.

Dreyfus, H.L., 2013, "The Myth of the Pervasiveness of the Mental", en Schear 2013b.

— , 2007a, "Response to McDowell", Inquiry, vol. 50, no. 4, pp. 371377.

— , 2007b, "The Return of the Myth of the Mental", Inquiry, vol. 50, no. 4 , pp. 352-365.

— , 2007c, "Detachment, Involvement, and Rationality: Are We Essentially Rational Animals?", Human Affairs, vol. 17, no. 2, pp. 101-109.

- 1972, What Computers Can't Do: A Critique of Artificial Intelligence, Harper \& Row, Nueva York.

Dreyfus, H. y S.E. Dreyfus, 1999, "The Challenge of Merleau Ponty's Phenomenology of Embodiment for Cognitive Science", en G. Weiss y H. Fern (eds.), Perspectives on Embodiment. The Intersections of Nature and Culture, Routledge, Nueva York/Londres.

Fridland, E., 2012, "Knowing-How: Problems and Considerations", European Journal of Philosophy, http://onlinelibrary.wiley.com/doi/10.1111/ ejop.12000/pdf [fecha de última consulta: 26/06/2013].

Gottlieb, G. 2011, "Unreflective Action and the Argument from Speed", Pacific Philosophical Quarterly, vol. 92, no. 3, pp. 338-362.

Hutto, D., 2012, "Exposing the Background: Deep and Local", en Radman 2012.

Kelly, S., 2000, "Grasping at Straws: Motor Intentionality and the Cognitive Science of Skilled Behavior", en M. Wrathall y J. Malpas (eds.), Heidegger, Coping, and Cognitive Science. Essays in Honor of Hubert Dreyfus, vol. 2., MIT Press, Cambridge, Mass./Londres.

— 2001, "The Non-Conceptual Content of Perceptual Experience: Situation Dependence and Fineness of Grain", Philosophy and Phenomenological Research, vol. 62, no. 3, pp. 601-608.

McDowell, J., 2013, "The Myth of the Mind as Detached", en Schear 2013b. , 2010, "What Is the Content of an Intention in Action?", Ratio, vol. 23 , no. 4 , pp. 415-532.

— 2009a, "Conceptual Capacities in Perception", en Having the World in View. Essays on Kant, Hegel, and Sellars", en Harvard University Press, Cambridge, Mass./Londres.

— 2009b, "Avoiding the Myth of the Given", en Having the World in View. Essays on Kant, Hegel, and Sellars, Harvard University Press, Cambridge, Mass./Londres.

—, 2007a, "Response to Dreyfus", Inquiry, vol. 50, no. 4, pp. 366-370.

— 2007b, "What Myth?", Inquiry, vol. 50, no. 4, pp. 338-351.

- 2000, Mind and World. With a New Introduction, Harvard University Press, Cambridge, Mass./Londres.

- 1994, "The Content of Perceptual Experience", The Philosophical Quarterly, vol. 44, no. 175, pp. 190-205. 
Montero, B., 2013, “A Dancer Reflects", en Schear 2013b.

Noë, A., 2013, "On Overintellectualizing the Intellect", en Schear 2013b.

— , 2005, "Against Intellectualism", Analysis, vol. 65, no. 4, pp. 278290.

Peacoke, C., 1998, "Nonconceptual Content Defended", Philosophy and Phenomenological Research, vol. 58, no. 2, pp. 381-388.

Pippin, R.B., 2013, "What is 'Conceptual Activity?" ", en Schear 2013b.

Radman, Z., 2012 (ed.), Knowing without Thinking. Mind, Action, Cognition and the Phenomenon of the Background, Palgrave, Nueva York.

Rietveld, E., 2010, "McDowell and Dreyfus on Unreflective Action", Inquiry, vol. 53, no. 2 pp. 183-207.

Rouse, J., 2013, "What is Conceptually Articulated Understanding?", en Schear 2013b.

Schear, J., 2013a, “Are We Essentially Rational Animals?", en Schear 2013b.

_, $2013 \mathrm{~b}$ (ed.), Mind, Reason and Being-in-the-World: The McDowell-Dreyfus Debate, Routledge, Londres.

Stanley, J., 2011, "Knowing (How)", Nous, vol. 45, no. 2, pp. 207-238.

Stanley, J. y T. Williamson, 2001, "Knowing How", The Journal of Philosophy, vol. 98, no. 8, pp. 411-444.

Travis, Ch., 2007, "Reason's Reach", European Journal of Philosophy, vol. 15 , no. 2 , pp. 225-248.

—_, 2004, "The Silence of the Senses", Mind, vol. 113, no. 449, pp. 5794.

Recibido el 26 de junio de 2013; revisado el 19 de diciembre de 2014; aceptado el 30 de abril de 2015. 